\section{Thin-Layer Chromatographic Separation of Copper, Nickel, Cobalt and Bismuth as Diethyl Dithiocarbamate Complexes}

Dünnschicht-chromatographische Trennung von Kupfer, Nickel, Kobalt und Wismut als Diäthyldithio-

carbamatkomplexe

Trenn. von Kupfer, Nickel, Kobalt, Wismut; Chromatographie, Dünnschicht; DDC-Komplexe

A. L. J. Rao and Surinder Singh

Chemistry Department, Punjabi University, Patiala-4, India

Received March 3, 1973

In the present study some of the metal diethyl dithiocarbamate complexes which are coloured and direct absorptiometric determination is possible have been separated on silica gel $G$ layers for which complete extraction data are available.

Experimental. Different aliquots of metal ion solutions are extracted with carbon tetrachloride containing 0.01 to $0.03 \mathrm{M}$ NaDDC at $\mathrm{pH} 6$.

Solvent systems used are

1. methylene dichloride/petroleum ether $\left(60-80^{\circ} \mathrm{C}\right)(5: 3)$;

2. methylene dichloride/cyclohexane (5:3). Running time is $20-30 \mathrm{~min}$.

Thin-layer chromatography was carried out on glass plates $(10 \times 20 \mathrm{~cm})$ coated with $250 \mu \mathrm{m}$ thick layers of silica gel $G$ according to Stahl on the air dried chromatoplates activated at $110-120^{\circ} \mathrm{C}$ for $30 \mathrm{~min} .10 \mu \mathrm{l}$ of the extracts were applied on a line $1.5 \mathrm{~cm}$ from the lower edge of the

\section{Zur dünnschicht-chromatographischen Trennung und Ermittlung der Nachweisgrenzen von Adrenalin und Noradrenalin an aktivierten Fertigfolien* mit unterschiedlichen Sprühreagentien}

Thin-Layer Chromatographic Separation and Determination of the Detection Limits of Adrenaline and Noradrenaline on Activated Ready-Made Foils using Various Spray Reagents

Trenn. von Adrenalin und Noradrenalin; Chromatographie, Dünnschicht; Fertigfolien

H. Thielemann

Eingegangen am 2. April 1973

Potter $u$. Mitarb. [6] trennten Adrenalin, Noradrenalin und einige Metaboliten dieser Verbindungen mit dem Fließ-

* Verwendet wurden Silufol Fertigfolien UV 254 der Fa. Sklárny Kavalier (ČSSR) mit folgender Zusammensetzung: Sorptionsmittel: Kieselgel für Chromatographie mit einem Luminescenzindicator; Unterlage: Aluminiumfolie; Bindemittel: Stärke.
Table 1. Rf $X 100$ values of metal-DDC complexes

\begin{tabular}{llll}
\hline Metal ion & Colour & \multicolumn{2}{c}{ Rf X 100 values in } \\
& & \multicolumn{2}{c}{ solvents } \\
& & 1 & 2 \\
\hline $\mathrm{Cu}$ & brown & 56 & 46.2 \\
$\mathrm{Ni}$ & yellow & 45 & 40 \\
$\mathrm{Co}$ & green & 27 & 30 \\
$\mathrm{Bi}$ & yellow & 21 & 22.3 \\
\hline
\end{tabular}

plates and $1.5 \mathrm{~cm}$ apart from each other. Complete separation was achieved in $20-30 \mathrm{~min}$. Results are presented in Table 1.

Interferences. Molybdate, tungstate, chromate, chromium and aluminium do not interfere with the extraction. Mercury, cadmium, lead, thallium and manganese are also extracted under similar conditions but being colourless do not interfere with the TLC separation of $\mathrm{Co}, \mathrm{Ni}, \mathrm{Bi}$ and $\mathrm{Cu}$.

Discussion. In this method the separated constituents can also be determined quantitatively by performing the TLC separation on the preparative scale, extracting the individual metal ion spots with carbon tetrachloride and measuring the colours with a spectrophotometer at the following wavelengths: Bi-DDC $366-370 \mathrm{~nm}$, Co-DDC $367 \mathrm{~nm}$, Ni-DDC $326 \mathrm{~nm}, \mathrm{Cu}-\mathrm{DDC} 436 \mathrm{~nm}$.

Dr. A. L. J. Rao

Chem. Dept.

Punjabi University

Patiala-4

India

mittel n-Butanol, das mit $3 \mathrm{~N} \mathrm{HCl}$ gesättigt war, auf Cellulose-Schichten mit Kammersättigung ( $6,5 \mathrm{~g}$ Cellulose $\mathrm{MN}-300$, $45 \mathrm{ml}$ Methanol; Schichten $10 \mathrm{~min}$ bei $105^{\circ} \mathrm{C}$ trocknen). Noradrenalin und Adrenalin wurden z.B. mit dem Fließmittel n-Butanol/Eisessig/Wasser $(40+10+50)$ ebenfalls auf Cellulose-Schichten (20 g MN-Cellulose $300 \mathrm{G}, 100 \mathrm{ml}$ Wasser; Schichten $2 \mathrm{~h}$ bei $100^{\circ} \mathrm{C}$ trocknen) chromatographiert. Bei Verwendung des genannten essigsauren FlieBmittels oder neutraler Fließmittel tritt beim Auftragen der Basensalze oder bei Anwesenheit stärkerer Säuren als der zur Fließmittelherstellung verwendeten, auf dem Startfleck die schon von der Papier-Chromatographie her bekannte Doppelfleckbildung auf; deren Ursache von Beckett $u$. Choulis [1] nochmals erörtert wurde.

Auf Fertigfolien ist die Doppelfleckbildung in geringem Umfang zu beobachten. Durch die in solchen Schichten vorhandenen Metall- und Schwermetallverunreinigungen wird jedoch die Oxydationsempfindlichkeit der Adrenalinabkömmlinge erhöht. Daher werden Adrenalin und Noradrenalin mit $10 \%$ igem Äthanol auf gepufferten KieselgelG-Schichten (Sörensen-Puffer 6,8), die unter Zusatz von Natriumhydrogenbisulfit hergestellt waren, chromatographiert [7].

Die Sympathomimetica und die durch Oxydation entstehenden Sekundärprodukte neigen außerdem zur Komplex- 